May 2017

\title{
Suicidal Ideation, Resilience, and Healthcare Implications for Newly Diagnosed HIV-Positive Men Who Have Sex with Men in China: A Qualitative Study
}

Haochu Li

Shandong University

Joseph Tucker

University of North Carolina at Chapel Hill

Eleanor Holroyd

Aga Khan University, eleanor.holroyd@aku.edu

Jie Zhang

Shandong University

Baofa Jiang

Shandong University

Follow this and additional works at: https://ecommons.aku.edu/eastafrica_fhs_sonam

Part of the Nursing Commons

\section{Recommended Citation}

Li, H., Tucker, J., Holroyd, E., Zhang, J., Jiang, B. (2017). Suicidal Ideation, Resilience, and Healthcare Implications for Newly Diagnosed HIV-Positive Men Who Have Sex with Men in China: A Qualitative Study. Archives of Sexual Behavior, 46(4), $1025-1034$.

Available at: https://ecommons.aku.edu/eastafrica_fhs_sonam/105 
Suicidal Ideation, Resilience, and Healthcare Implications for Newly Diagnosed HIV-Positive Men Who Have Sex with Men in China: A Qualitative Study

\section{Haochu Li, Joseph Tucker, Eleanor Holroyd, Jie Zhang \& Baofa Jiang}

\section{Archives of Sexual Behavior}

The Official Publication of the

International Academy of Sex Research

ISSN 0004-0002

Arch Sex Behav

DOI 10.1007/s10508-016-0894-0

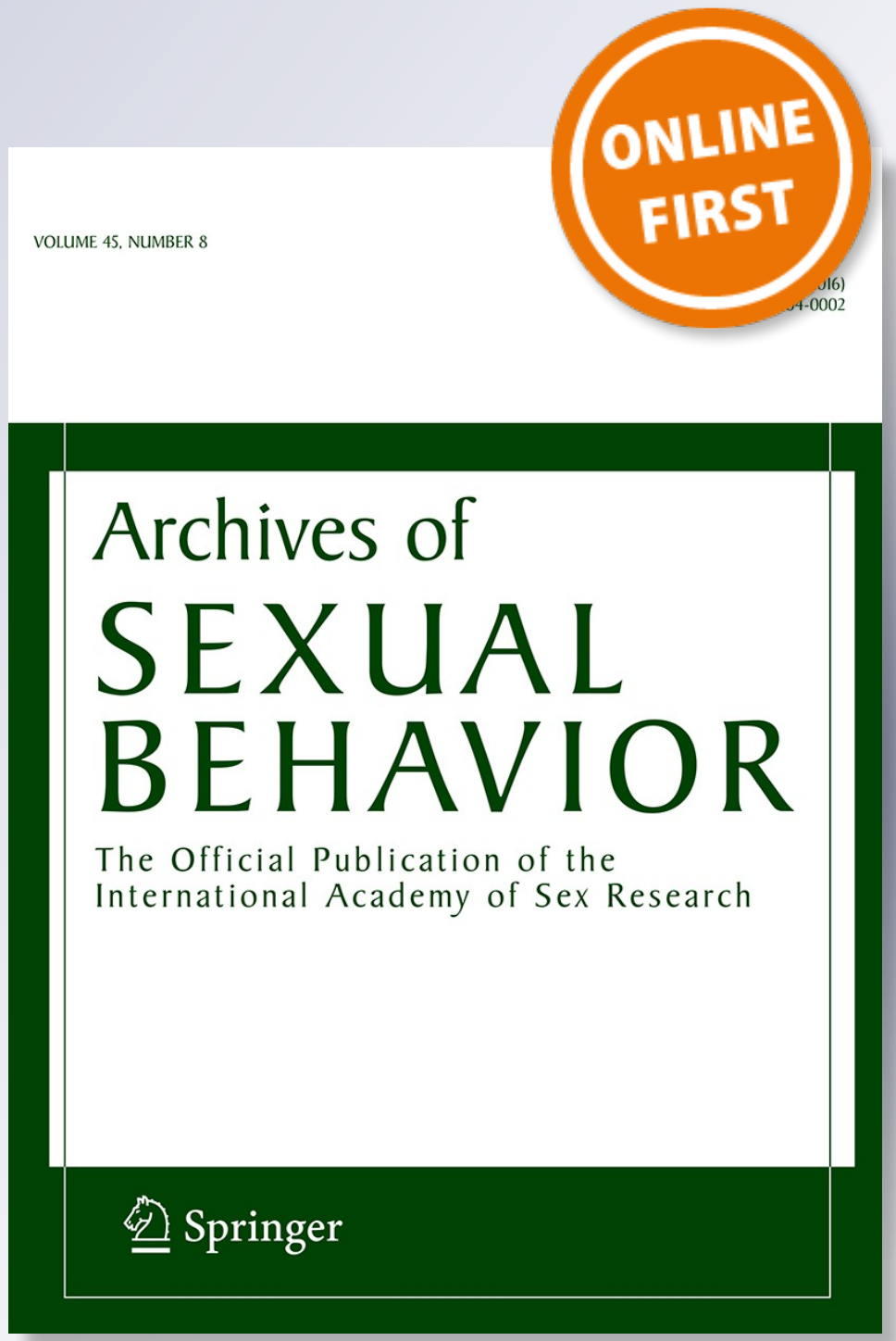

Springer 
Your article is protected by copyright and all rights are held exclusively by Springer Science +Business Media New York. This e-offprint is for personal use only and shall not be selfarchived in electronic repositories. If you wish to self-archive your article, please use the accepted manuscript version for posting on your own website. You may further deposit the accepted manuscript version in any repository, provided it is only made publicly available 12 months after official publication or later and provided acknowledgement is given to the original source of publication and a link is inserted to the published article on Springer's website. The link must be accompanied by the following text: "The final publication is available at link.springer.com". 


\title{
Suicidal Ideation, Resilience, and Healthcare Implications for Newly Diagnosed HIV-Positive Men Who Have Sex with Men in China: A Qualitative Study
}

\author{
Haochu $\mathrm{Li}^{1,2} \cdot$ Joseph Tucker $^{2}$ Eleanor Holroyd ${ }^{3,4} \cdot$ Jie Zhang $^{1,5} \cdot$ Baofa Jiang1 $^{1}$
}

Received: 19 February 2016/ Revised: 17 October 2016/ Accepted: 26 October 2016

(C) Springer Science+Business Media New York 2016

\begin{abstract}
Globally, suicidal ideation and behavior have been widely reported among people living with human immunodeficiency virus (HIV). Knowledge gaps exist regarding the longer life and resilience-related experiences of people living with HIV (PLWH). Specifically, there is a dearth of data about the interaction of perceived risk and resilient factors resulting in a wide spectrum of intentional suicidal ideation outcomes in a Chinese cultural context. This qualitative research drew from a larger ethnographic study of newly diagnosed HIV-positive men who have sex with men (MSM) in Mainland China. Interviews were conducted with 31 MSM within 6 months of diagnosis of HIV infection. Initial suicidal ideation was commonly reported with participants subsequently feeling more resilient to these thoughts through gaining a greater understanding of their prognosis and treatment. Post-HIV diagnosis, some participants reported forming new relationships and receiving increased support from their partners, friends, peers, families, and community-based organizations. At follow-up, these participants generally reported suicidal ideation had declined. However, participants who continued to express suicidal ideation perceived extended pressure from their families' expectations for them
\end{abstract}

Haochu Li

haochuli@med.unc.edu; hcli@alumni.cuhk.net

1 School of Public Health, Shandong University, No. 44 Wen Hua Xi Road, Jinan 250012, China

2 UNC Project-China, Institute for Global Health and Infectious Diseases, University of North Carolina at Chapel Hill, Chapel Hill, NC, USA

3 School of Clinical Sciences, Auckland University of Technology, Northcote, Auckland, New Zealand

4 School of Global and Population Health, The University of Melbourne, Melbourne, Australia

5 Department of Sociology, State University of New York College at Buffalo, Buffalo, NY, USA to engage in heterosexual marriages and parenthood. Furthermore, these men reported ongoing hardships in their daily life, unemployment, lack of social support, and isolation. Among this Mainland Chinese cohort of HIV-positive MSM, suicidal ideation may be a transient phenomenon experienced initially following HIV diagnosis that resolves with increased and specific familial, social, and service-based support. It is crucial to identify the causes of stress and social suffering associated with HIV diagnosis in order to reduce suicidal ideation. In China, action is needed to develop routine mental health screening and to increase services that support PLWH. Important services mechanism to accomplish this are promoting resilience through intentional activities as well as continued public health campaigns to reduce stigma toward HIVpositive MSM.

Keywords Suicidal ideation · Resilience $\cdot$ HIV . Sexual orientation $\cdot$ Men who have sex with men . China

\section{Introduction}

Suicidal ideation, concerning thoughts about or preoccupation with suicide, has long been associated with HIV infection (Perry, Jacobsberg, \& Fishman, 1990; Starace \& Sherr, 1998). A systematic review showed that $28.5 \%$ of people living with HIV (PLWH) reported suicidal ideation during the preceding week (Catalan et al., 2011). Likewise, a study from the U.S. reported that approximately $28 \%$ HIV-infected men who have sex with men (MSM) reported suicidal thoughts in the preceding 2 weeks post-diagnosis (Carrico, Neilands, \& Johnson, 2010). In the UK, there was a $31 \%$ prevalence of suicidal ideation among HIV clinic attendees (Sherr et al., 2008).

Increased effectiveness and access to antiretroviral treatment (ART), PLWH's overall health status has improved with suicide 
rates among PLWH being correspondingly declined; however, the rates remain more than three times higher than those in the general population (Keiser et al., 2010). It is well documented that suicidal ideation may precede suicidal acts, such as planning and attempting suicide (Kessler, Borges, \& Walters, 1999). The association between elevated suicidal ideation and increased HIV transmission risk has been reported among PLWH (Carrico et al., 2007) and MSM populations (Carrico et al., 2010).

Suicidal ideation is a substantial indicator of future mental health burden (Hawton, Rodham, Evans, \& Weatherall, 2002). It is important to monitor suicidal ideation and respond to it appropriately as a key strategy in national suicide prevention programmes, thus improving health outcomes and quality of life among PLWH.

\section{Suicidal Ideation and HIV Infection}

Among PLWH, studies have explored factors associated with suicidality. Individual risk factors for suicidal ideation and behavior include: stressful life events/trauma (Plena, Singer, \& Goldbeck, 2011), withdrawal/isolation from friends and family, physical illness or injury (Christiansen \& Stenager, 2009; Riala, Ilomaki, Hakko, \& Rasanen, 2011), sexual or other abuse (Weich, Patterson, Shaw, \& Stewart-Brown, 2009), drug and alcohol misuse/dependency (Wilcox, Conner, \& Caine, 2004), depression and anxiety (Capron, Gonzalez, Parent, Zvolensky, \& Schmidt, 2012; Gibbs et al., 2009; Phillips, 2010), and lack of disclosure of HIV status and ceasing ART (Sherr et al., 2008). Interpersonal risk factors for suicidal ideation and behavior include: relationship breakups and domestic violence (Devries et al., 2011), homelessness, torture or trauma survival, reluctance to seek help (Francis, Pirkis, Dunt, Blood, \& Davis, 2002), financial difficulties (McNamara, 2013), and managing negative family and community responses to same sex attraction (Pitts, Smith, Mitchell, \& Patel, 2006; Ryan, Huebner, Diaz, \& Sanchez, 2009). Socioeconomic risk factors for suicidal ideation and behavior have been reported as: financial difficulties, unemployment (Scutella \& Wooden, 2008), housing uncertainty and/orhomelessness, social isolation, lack of community support services (Cattell, 2001), and a culture of reluctance to engage in help seeking within communities (McNamara, 2013). It has additionally been reported that some local communities manifest suicide clustering and an element of contagion (Hanssens, 2010).

In China, suicide often occurs without prior diagnosis of mental illness or disorders. Approximately $63 \%$ of completed suicides in China had previously reported symptoms of mental disorders such as anxiety or depression (Phillips, Li, \& Zhang, 2002). This is considerably less than what is reported in most Western countries where the estimates are as high as $90 \%$ (Bertolote, Fleischmann, De Leo, \& Wasserman, 2003; Lesage et al., 1994). The indication is that while suicide and depression can result from the same familial conflicts, they are not necessarily causally linked to them (Phillips et al., 2002).

In China, suicidal ideation has been identified as a common psychological characteristic in HIV-infected MSM. This may result from psychological pressure attributed to a lack of acceptance by mainstream society (Chen et al., 2012), feelings of being overwhelmed, physical pain that is uncontrolled, and financial difficulties arising from healthcare expenditures $(\mathrm{Li}, \mathrm{Xiao}, \&$ Xiao, 2009).

\section{Resilience to Suicidal Ideation in the Context of HIV Infection}

Resilience is defined as a collection of negative life events resulting in positive outcomes (Masten, 2001). Studies have described suicide resilience as a set of beliefs or perceptions which buffer individuals against the development of suicidal ideation in the face of accompanying risk factors (Johnson, Gooding, Wood, \& Tarrier, 2010; Osman et al., 2004; Rutter, Freedenthal, \& Osman, 2008). Suicide resilience is intended to reduce potential suicidal ideation and behavior (Johnson, Wood, Gooding, Taylor, \& Tarrier, 2011). Suicide risk and resilience can be viewed as opposite ends of a spectrum, and understanding the interaction of these two domains is of vital importance (Johnson et al., 2011).

A study of MSM with AIDS showed that participants who were resilient to suicidal ideation reported having a support system, consistent medical care, and used psychotherapy services to improve coping strategies (Rabkin, Remien, Katoff, \& Williams, 1993). It was reported that participants who were more resilient were able to tolerate the uncertainty associated with HIV infection, were able to remain in employment, and were intending to form intimate relationships with others (Thompson, 2002). A case study by Bletzer (2007) showed that diagnosis of HIV infection pushed participants to make positive life changes. The initial diagnosis was disruptive, but through improved HIV knowledge, reconnection to cultural backgrounds, selfresponsibility, persistence, control, and a focus on presentday events, participants were able to reconnect with their community and to access healthcare resources to help them manage the illness. Another case study of MSM in the final stages of AIDS showed that despite the obstacles of a stigmatized illness and a marginalized life, those interviewed were empowered by community support that promoted illness adaptation, and through this, they were able to progress through the end stages of their illness with their last wishes respected (Cadell, Karabanow, $\&$ Sanchez, 2001).

\section{Research Aims}

It has been reported that HIV diagnosis greatly increases the risk of suicidal ideation and behavior (Meel \& Leenaars, 2005). Siegel and Meyer (1999) found that people generally experienced 
terror and confusion when first notified of being HIV-positive, such as frightening images of a future life with AIDS. Even though suicidal ideation may decline over time following diagnosis of HIV infection (Samuelsson, Wiklander, Asberg, \& Saveman, 2000), not all PLWH were able to move through this adaptive process. There remains a knowledge gap in the long-term factors that promote resilience among PLWH. With specific regard to the Chinese cultural context, any analysis of risk and resilience requires consideration of cultural influences including: obligations to be married, obligations for parenthood and continuing the family name, engaging in filial piety (e.g., providing financial support to families), and protecting the family reputation (Li, Holroyd, Lau, \& Li, 2015b). Few studies have examined suicidal ideation in relation to the dynamic interplays of risk and resilience in the context of HIV-infected MSM, and to date no identified studies have comprehensively examined this within a Chinese cultural context. The following study therefore aims to examine the psychosocial context immediately following new diagnoses of HIV among MSM, and to follow them longitudinally to explore coping and resilience among this group. The results will be used to inform HIV-related healthcare services provision and support for PLWH in Mainland China.

\section{Method}

\section{Participants}

This research was conducted in the city of Shenzhen, China. In 2010 , Shenzhen had a population of 13.1 million and more than $80 \%$ of this population was domestic migrants (Shenzhen City Government General Office, 2011). It was estimated that 100,000-200,000 MSM were living in Shenzhen in 2009 (Xie et al., 2010). Repeated in-depth interviews (an initial interview and a follow-up interview conducted at 3-month intervals) were undertaken from January to September 2010, in collaboration with a local non-government organization (NGO). The nominated NGO worked closely with the Shenzhen Centre for Disease Control and Prevention to provide HIV prevention services (e.g., free condoms, lubricant, HIV testing, and hotline) to MSM and was well connected to grass-root support groups for PLWH. A life profile approach was employed, undertaking background research, lengthy interviews, and close collaborations with participants to ensure that testimonies accurately reflected the degree of depth related to the life-world or social themes. The life profile enabled a focus on individual life narratives that provided background and context to an individual's contemporary community life (McCance, McKenna, \& Boore, 2001). A semi-structured interview guide was developed based on an initial literature review which included the following major questions: After your HIV diagnosis, how did you feel and what thoughts did you have? What did you do to respond to it and/or adjust? How did others react to you? And how did you deal with their reactions?

Purposive sampling was used to recruit participants from diverse backgrounds with a range of characteristics. Full details are listed in Table 1. Participants were eligible for inclusion in the study if: they were 18 years of age or older, received a diagnosis of HIV infection within the last 6 months (newly diagnosed), identified as a man whohas sex with men, and was willing and able toprovide written informed consent. In order to capture detailed information of adaptation to the new HIV diagnosis, a total of 31 eligible participants completed the repeated in-depth interviews. All participants were internal migrants from other provinces/prefectures/ regions of Mainland China.

Table 1 Summary of the characteristics of participants (newly diagnosed HIV-infected men who have sex with men; $N=31$ )

\begin{tabular}{|c|c|}
\hline Characteristics & $N(\%)$ \\
\hline \multicolumn{2}{|l|}{ Age (in years) } \\
\hline $18-25$ & $8(25.81)$ \\
\hline $26-35$ & $19(61.29)$ \\
\hline $36-40$ & $4(12.90)$ \\
\hline \multicolumn{2}{|c|}{ Duration since diagnosis (months) } \\
\hline $1-2$ & $15(48.39)$ \\
\hline $3-4$ & $11(35.49)$ \\
\hline $5-6$ & $5(16.13)$ \\
\hline \multicolumn{2}{|l|}{ Occupation } \\
\hline Office & $10(32.26)$ \\
\hline Service/sales & $5(16.13)$ \\
\hline Technician & $9(29.03)$ \\
\hline Laborer & $4(12.90)$ \\
\hline Sex worker & $1(3.23)$ \\
\hline Jobless & $2(6.45)$ \\
\hline \multicolumn{2}{|l|}{ Education } \\
\hline College & $8(25.81)$ \\
\hline High/technological school & $17(54.84)$ \\
\hline Secondary school & $6(19.35)$ \\
\hline \multicolumn{2}{|l|}{ Monthly income (RMB) } \\
\hline More than 6000 & $3(9.68)$ \\
\hline $3000-6000$ & $12(38.71)$ \\
\hline Less than 3000 & $14(45.16)$ \\
\hline No income & $2(6.45)$ \\
\hline \multicolumn{2}{|l|}{ Sexual identity } \\
\hline Homosexual & $28(90.32)$ \\
\hline Bisexual & $3(9.68)$ \\
\hline Married & $5(16.13)$ \\
\hline On ART & $8(25.81)$ \\
\hline
\end{tabular}

$\overline{R M B}$ Renminbi yuan, \$1 U.S. $=6.80 \mathrm{RMB}$ in 2010; $A R T$ antiretroviral therapy 


\section{Procedure}

Ethics approval was granted by the Survey and Behavioral Research Ethics Committee at the Chinese University of Hong Kong. The nominated NGO contacted potential participants through their networks according to the study's purposive sampling schema. Participants were informed that refusal would not affect their right to use services and that the content of the interview would be digitally recorded. Written informed consent was obtained. The men interviewed were assured about data confidentiality, use of pseudonyms, safe storage of the data, and the right to withdraw at any stage without prejudice. The follow-up interviews were conducted 3 months after the initial interviews. The initial interview was transcribed verbatim, and emerging themes were recorded during the analysis. A constant comparative analysis enabled the subsequent interview guide to develop further questions, or to explore incomplete information in the second interview. Each interview lasted approximately one and a half to $2 \mathrm{~h}$ and was conducted in a private room in the NGO's office. Participants received RMB 500 (approximately \$80USD) in cash after completion of the repeated interviews as compensation for their time and travel costs.

\section{Data Analysis}

The interviews were conducted in Mandarin Chinese, and taperecorded interviews were transcribed verbatim at the completion of each interview. Thematic content analysis was used concurrently with data collection in order to capture emerging themes (Green \& Thorogood, 2009). Participants who expressed thoughts to endanger or end their lives were identified as having suicidal ideation. In subsequent twice-weekly meetings, areas of emerging themes and data saturation were discussed among the research team and agreement was reached on a set of preliminary codes. The later in-depth analysis first involved preliminary reading of all the transcripts, noting initial reactions and assigning preliminary thematic codes. The first author undertook all coding, which was duly discussed with co-authors to reach member agreement. The transcribed interviews, notes, memos, codes, and decision-making were kept for audit trail (Rodgers \& Cowles, 1993). Suicidal ideations were identified from the narratives and were coded with the assistance of a senior psychiatrist with a specialist background in HIV infection in MSM. Related psychosocial and cultural factors emerging from participants' reflections on their suicidal ideations were also thematically coded, such as cognition of HIV/AIDS (a mental process involving knowing, learning, and understanding HIV/AIDS), financial difficulty, family and social support, social norms, coping and resilience. Informative quotes relevant to the themes were grouped together and later synthesized. Thematic structures based on collective discussion was further refined. The first author translated the transcripts into English, and the co-authors cross-checked the accuracy and completeness of translations.

\section{Results}

Among the total 31 participants, most men were aged 26-35 years $(61.29 \%)$. The majority of participants $(51.61 \%)$ had a monthly income of less than RMB 3000 (USD 441). Most of the participants $(74.19 \%)$ had an education level of high scholar diploma/technology apprenticeship or less. Most of the participants identified as homosexual (90.32\%), and some participants reported being married (16.13\%). Participants had been diagnosed with HIV in the following time periods: $48.39 \%$ in 1-2 months, $35.49 \%$ in $3-4$ months, and $16.13 \%$ in $5-6$ months. Only $25.81 \%$ of the participants reported actively taking ART. After diagnosis of HIV infection, some participants (14/31) expressed a range of degrees of suicidal ideation. Some participants merely mentioned their wish to die, and others elaborated on their reasons for wanting to commit suicide and described their plan to do so. Factors associated with suicidal ideation among this cohort are presented below.

\section{Negative Cognition of HIV/AIDS}

Many participants reported very high levels of distress upon a diagnosis of HIV and felt a variety of negative and catastrophic thoughts. Some participants expressed anxiety about physical symptoms that they perceived to be connected to HIV infection (e.g., having red dots over body as an opportunistic infection, or getting very thin). Participants were fearful that their HIV infection and the advancing disease progression to AIDS would bring a difficult and painful death. Participants considered suicide as an available option to avoid the physical consequences that would accompany HIV infection. A few participants also reported denial, disbelief, and difficulty in accepting their HIV diagnosis. Even though they didn't feel any illness related to HIV at the time of diagnosis, they testified to feeling that they were "having no way out" and "wanting to die sooner and not having a long drawn out death."

Kong (aged 26, a service worker, RMB 800 per month) said:

I am not afraid of death. If I were to kill myself, it would not bring me too much suffering. I thought it is better for me to die, and then I would not think about this thing [HIV/AIDS]....For example, that day I accidentally saw on the Internet images of people dead or dying from AIDS ...it was too horrible to look at; they were completely emaciated. I found it unbearable and felt awful. If this should happen to my body I would not know what to do or how to accept that it was happening to me.... I found myself thinking that one day this will be me...I felt really terrible. I couldn't bear thinking about it.

Kong's case showed that HIV/AIDS was perceived as very negative and catastrophic. The appearance of PLWH's body 
was perceived as emaciated and awful. This horrible HIV-related image and their anticipating consequences became a very heavy psychological burden that resulted in suicidal ideation.

\section{Financial Difficulty}

Some participants reported financial difficulties. Their experience of living with HIV created further financial problems that have negatively impacted upon theirquality of life. Hei (aged 25, a laborer, RMB 1500 per month) reported "I have this illness [probably a kind of opportunistic infection], but I cannot afford to go to hospital for treatment." In China, HIV testing and ART are provided free-of-charge by government health centers. However, PLWH are required to pay a fee-for-service charge for medical checkups and treatment of opportunistic infections. HIV infection and financial difficulties were reported to encourage gambling among a few participants in an attempt to obtain financial security. Xie (aged 31, an office worker, RMB 2500 per month) reported:

I took my chances at a casino. If I won hundreds of thousand, I do not want to go to work under the pressure of this disease. But in fact, I lost more than ten thousand. That was all the money I had saved for a year....I was devastated...I wanted to jump in the river.

Wei (aged 26, a technician, RMB 5000 per month) said:

One day I got a headache and went to a hospital for a comprehensive medical examination. I met with some patients on the ward. They needed continuous medical treatment but they had neither medical insurance nor money. They suffered from many diseases. It had a big impact on me. I started my business. I decided to go all out at work. I thought even though I have HIV, if I work hard and am successful, I do not need to worry about the cost of my medical treatment in the future....I do not want to burden my family. If I fail in my business, which as you know, in our society, is a very real possibility, I would rather die than have to live with such chaos and uncertainty.

Wei's case highlights how witnessing others living with HIV and encountering financial difficulties as a result of medical treatments increased determination to work and be successful. Participants who saw others suffering from HIV/AIDS felt that this prompted the ideation that suicide could be a viable option.

\section{Lack of Family and Social Support}

A lack of social support was widely related to suicidal ideation among participants. Some reported that the loss of significant others negatively impacted upon their ability to cope and contributed to feelings of despair. A participant (Qi, aged 35, an office worker, RMB 2500 per month) said, "Because of this issue [end of a romantic relationship], I attempted suicide....I do not believe in love anymore." Participants who reported not having friends or partners also reported feeling hopeless and isolated. A few participants even reported they felt apathy from their families. For example, Hei (aged 25, a laborer, RMB 1500 per month) said, "My families were very apathetic....My mother would not give me money to go to hospital. I was full of despair....I really want to die."

Xie (aged 31, an office worker, RMB 2500 per month) said:

I do not have a partner. I feel it is meaningless to live.... when I see other people in relationships, I feel my life is over. I attempted suicide once during that time.... What was worse was my skin disease. Ioften get allergies and grow red spots, which makes it difficult for me to make friends. Otherpeople would think that I have some infectious disease. Actually it is a kind of Psoriasis, and it is genetic.... Others can live in the dorms happily, chatting and having fun. But I cannot. Because of my body [HIV infection] and skin disease, I have to rent a room outside now.

Xie's case as outlined above indicated that the absence of a partner (romantic relationship) made his life meaningless and despairing. The diagnosis of HIV combined with the public visibility of his skin disease resulted in feelings of isolation and exclusion.

\section{Suicidal Ideation as a Common Response to HIV}

Some participants reported that suicide was often discussed on Internet chat rooms and through social media, such as QQ groups [QQ is a major social networking platform heavily used by MSM in China]. Xie reported that, on the Internet, he has observed "Many people could not bear this stress and committed suicide." This observation made him feel "very disappointed." Hei shared his personal experience online, stating "Sometimes when we [PLWH in QQ groups] were in low spirits, we would say 'let us die together.... we will go together and jump to the sea' or 'together we can run into traffic'." A few participants were reflective and noted how their suicidal ideation may influence other PLWH.

Feng (aged 31, a technician, RMB 3000 per month) said:

Previously I would like to describe how I was feeling in my QQ signature....I described my feelings about taking ART ....I wanted to give up at that time and I wrote in my QQ signature that "ART is terrible. I really want to give up."

Some people were curious to ask me questions. I suddenly realized that my attitude would affect others.

Feng's case indicated that participants' negative comments of taking ART may influence other PLWH's perceptions of their own outcomes. Suicidal ideations among PLWH are widely communicated on social media and may be perceived as a common response to HIV in these specific communities. 


\section{Factors Promoting Resilience}

Most participants (12/14) who reported suicidal ideation in the first interviews did not report this again in the subsequent interviews. Negative perceptions of HIV infection and AIDS were reported to be "gradually" disappearing. This changing process may be attributed to several reasons.

First, many participants reported positive encounters with AIDS activists, other PLWH, volunteers, and peers. At the beginning of their diagnosis, many participants feared rapidly dying from HIV, but at follow-up 3 months later, most of them stated that they would not die immediately. At this point some participants reported experiencing a type of psychological buffer in that while they may die of AIDS or its complications, this would not occur until further into the future. Han (aged 25 , an office worker, RMB 2000 per month) reported:

I surfed the Internet to search [HIV/AIDS] related information, having more understanding....[Other PLWH] shared their experiences and ideology changing....I communicated with volunteers....Gradually, I realized that the status of getting HIV positive changed nothing, and I still need to work and have life.

These participants also reported a sense of hope that came from advances in HIV/AIDS medication and treatment.

Second, the practices of intentional activities (i.e., intentionally engaging in activities that tackle problems or issues in one's life) appear as important factors promoting resilience from suicidal ideation. Shi (aged 26, an office worker, RMB 3000 per month, married, bisexual) reports:

I went to be a volunteer, getting to know more about PLWH, searching the Internet, and listening to the hearts of other PLWH....getting along well with them and taking it easier." Han also reported "I asked for a leave for a week. . .having a rest, I gradually adjusted myself....I became more serious about my job.... Once I get sick, the treatments need a lot of money.

Participants commonly intentionally adjusted their emotions and attitudes toward their HIV diagnosis.

Third, participants described how social and psychological support and family responsibility had increased their ability to resist suicidal ideation. For example, Zhu (aged 27, an office worker, RMB 4000 per month) said, "If I die, how my mum can survive? I told myself that I should not think about it [suicide] anymore....my parents were getting old....I need to think about my families." Shi also noted, "Since I have a child, I don't think about other issues. I just want to earn more money for my child....I don't give up my life." Shi further described, "I told my wife [about HIV positive, but not having sex with men], she is still willing to live with me and she accepts this reality....She treats me really well."

Fourth, romantic relationships (e.g., having a stable partner, orfalling in love) provided important support structures and resulted in increased resilience among a few participants. Developing a relationship facilitated emotional attachment and a positive view about life and the future. For example, having a partner encouraged Zhu to have a positive outlook on life. He said, "I feel I should do a good job in my work, working hard, playing hard, and releasing pressure....my emotional life is relatively stable and plain." Shi reports, "I met with a friend (a man). I fell in love with him.... anyway, I never had a feeling like that, just love him so much, and want to do anything for him.... we have phone contact four times per week at least, and meet one time per week....I really hope new drugs can come out soon." Falling in love has helped participants to be altruistic and feel greater optimism about future.

Resilience among newly diagnosed HIV-infected MSM is illustrated in the following example, where increased knowledge of HIV/AIDS disease progression and positive role models online contributed to the acceptance of HIV diagnosis. Hei said:

I am affected by other people living with HIV, such as XX [AIDS activists]. I read reports about them....Gradually, I get to know that I should live stronger, treat myself better, and maintain a better mentality. The red dots in my body get better gradually. I get to know more about AIDS, and get to know that people would not die immediately after getting HIV infection. It's a chronic disease.... This is a process of growing up, I think.

Hei's case indicates that when participants differed in their suicidal ideation, they were seen to reduce their feeling of uncertainty and hopelessness. After releasing this burden, they felt they were able to return to their normal lives, such as actively working, being happy with friends and colleagues, and even helping other newly diagnosed PLWH.

\section{Factors Influencing Sustained Suicidal Ideation}

Only two participants (Qian and Shen) reported suicidal ideation at baseline and follow-up. Qian (aged 25, a technician, RMB 2000 per month) reported his suicidal ideation stemmed from family beliefs about sexuality and pressure to engage in heterosexual marriage. Qian said:

I got to know about a boyfriend who is also a person living with HIV. After we are together, we do a brave thing, coming out of closet to my families.... But they (families) all think that I am sick and push me to see a doctor....my families introduce girlfriends to me and I feel very anxious these days. I don't know what to do....they got angry and called me frequently. I got very angry and suffered so much....I don't have the mood to work at all. Somebody always called me no matter sleeping or working. So much pressure and anxiety!

Qian reported that his sexual preference was unacceptable to his family and that this resulted in increased anxiety, having difficulty sleeping and experiencing low mood. 
Shen (aged 29, unemployed) reported that his suicidal ideation stemmed from unemployment and psychosocial problems (e.g., low mood, lack of social support, and isolation). Shen said:

I don't have any families or relatives in Shenzhen, basically no friend....I don't have a job now, not in a good mood. I don't know what to do. It's really bothersome to think about this issue....I now cannot fall asleep, waking up at one or two a clock at night. But during the day time, I am sleepy, and feel especially exhausted and bored.... getting sick...I feel great pressure.... I now feel so guilty to my parents.... I don't have friends, basically in isolation. ...I want to revenge the one who transmitted the virus to me, and then I commit suicide. I don't want to take medicine anymore. I complain joblessness, or pressure, that kind of stuff.

Shen reports that unemployment and lack of social support have resulted in confusion and fear about his future. He reports feeling depressed, having difficulty sleeping and sensing a desire for revenge.

\section{Discussion}

The association between suicidal ideation and diagnosis of HIV infection has been well documented in the literature. The majority of studies have focused on exploring and identifying risk factors related to suicidal ideation in the context of HIV infection. Even though the role of resilience in suicidal ideation has increasingly attracted attention, studies exploring the interaction between suicidal ideation and resilience among PLWH are limited. The current study adopted a longitudinal and dynamic approach within the Chinese cultural context. This examination of HIVinfected MSM in Mainland China found that most participants who had suicidal ideation early after their HIV diagnosis, later expressed that this ideation had diminished due in part to exercising some forms of resilience. In a specific Chinese context, supporting Durkheim's (1951) argument, suicidal risk is not simply a mental health concern, but an extension of different idiosyncratic social reasons to individuals. These social reasons included the cultural imperatives of heterosexual marriage, parenthood, and loss of face.

The current study explored a cohort of Chinese HIV-infected MSM's perceptions of suicidal ideation and ways of coping. The factors associated with suicidal ideation in this cohort included: negative cognition of HIV/AIDS, financial difficulties, lack of family and social support, and Chinese social norms (i.e., heterosexual marriage and parenthood). These findings compare with a study by Siegel and Meyer (1999) where men redefined HIV infection as chronic illness rather than acute once they had increased their knowledge and felt prepared for the slow progression of the disease. Their resilience to HIV was further established after obtaining social support from their families, friends, AIDS activists, vol- unteers, and NGOs and a sense of hope for more advanced medications or treatments. Integration of HIV into one's daily life was also reported through emotion regulation and having a more positive outlook of life with HIV (e.g., making future life plans despite the presence of a chronic illness). When the resilience end of the suicide ideation spectrum is perceived to have more impact than the risk end, suicidal ideation tends to diminish or disappear (Johnson et al., 2011).

Most of the participants who reported suicidal ideation testified to a degree of adjustment over time; however, some factors were repeatedly related to lead to suicidal ideation. These factors included: pressure to engage in heterosexual marriages, financial difficulties, lack of social support, and isolation. The diagnosis of HIV and MSM sexual practices confronted Chinese cultural norms, particularly heterosexual marriage and maintaining morality (Li, Holroyd, \& Lau, 2010a). In the Chinese context, lian (face, moral position) and mianzi (face as self-affirming social status) are key moral resources (Kleinman \& Kleinman, 1997). MSM who are diagnosed with HIV in China must contend with these expectations for maintaining face and morality.

In China it has been reported that PLWH are socially considered to have lead immoral lives (Wu, 2009). A person with HIV/AIDS constitutes not only strictly an individual concern, but also a societal concern, limiting Chinese family bloodlines. Such cultural contagions can result in suicide outcomes, violating the cultural requirement of being a moral citizen in a Chinese context (Wu, 2011). MSM commonly experience difficulties finding supportive relationships, particularly when diagnosed as HIV-positive, due to ostracism and stigmatization (Li, Holroyd, \& Lau, 2015a). In this regard, HIV/AIDS prevention and care services in China should not only focus on immediate medical care, but also target public moral acceptance and emotionally supportive service delivery. For example, the formal embedding protocols for respect in healthcare services provision could enable PLWH to have symbolic outcomes, such as hope.

This research yields important recommendations for addressing suicidal ideation among newly diagnosed HIV-infected MSM in Mainland China. First, it is imperative that mental health screening and counseling within the HIV/AIDS testing regime and follow-up services enable healthcare providers to tailor healthcare provision to the individual needs of each client. Psychological support and/or counseling at the time of diagnosis could reduce rates of suicidal ideation and improve resilience among vulnerable groups of HIV-infected persons in Mainland China. The continued monitoring of suicidal ideation and behavior, including treatment of neuropsychological deficits, should be a routine component of clinical and community health care among newly diagnosed HIV-infected MSM (van Heeringen, Bijttebier, \& Godfrin, 2011).

Second, it has been argued that intentional activities such as volunteerism and improved emotion regulation could help improve resilience among HIV-infected persons (Fredrickson, 2000; Smith, McCarragher, \& Brown, 2015). Intentional activ- 
ities have been shown to enhance well-being and to decrease depressive symptoms (Sin \& Lyubomirsky, 2009). Of particular interest is research showing that intentional activity changes have greater success at enhancing well-being, than do changes in circumstance (Sheldon \& Lyubomirsky, 2006). The participants of this research reported important intentional activities, such as volunteerism and improved emotion regulation. Research seeking to gain greater understanding of efficacy of intentional activity interventions, specifically among this cohort, has the potential to increase resilience and improve well-being and overall quality of life outcomes among HIV-infected MSM in Mainland China.

Third, participants' suicidal ideation is associated with Chinese cultural norms and family obligations, including pressure to engage in heterosexual marriage and to maintain morality. This pressure forms the social and cultural origins of stigma against HIV-infected MSM. Boulay, Tweedie, and Fiagbey (2008) encouraged using mass media campaigns to urge greater compassion for persons infected with HIV so as to reduce societal stigma toward PLWH. Likewise, in China, an intervention trial study targeting community leaders achieved significant HIV stigma reduction (Li, Liang, Lin, Wu, \& Rotheram-Borus, 2010b). Interventions designed to empower communities of PLWH, which also included financial support, have been shown to be effective at increasing community interactions between PLWH and those without HIV in Thailand (Apinundecha, Laohasiriwong, Cameron, \& Lim, 2007).

All effort was taken to ensure methodological rigor in this research; however, the study contains a number of limitations. First, it is possible that the qualitative in-depthinterviews created a space for participants to talk about suicidal ideation and an unintended intervention effect may have been resulted. Second, the use of a digital recorder to document the interviews may have prohibited some participants from talking openly about their views on contemporary Chinese society, particularly if their views were negative. Third, recruitment bias may also affect our results as only participants who had contact with healthcare workers or volunteers could be invited to participate. Fourth, this research focused on factors leading to suicidal ideation and those that fostered resilience among a group of newly diagnosed HIV-infected MSM in Mainland China. Analysis of suicidal ideation was further limited by lack of standardized scales to measure suicidal ideation. The sample size was small and not representative of all MSM groups within Chinese society. The results may not be representation of all HIV-infected MSM in Shenzhen or other parts of China.

\section{Conclusion}

This research reports on the experiences of suicidal ideation and resilience among a cohort of newly diagnosed HIV-infected MSM in Mainland China. Participants reported the need to negotiate the challenges of social and cultural norms, specifically expectations for heterosexual marriage and morality. Participants who displayed resilience to suicidal ideation and behavior reported exter- nal sources of support and sound understanding of the HIV disease progression process. These findings suggest that among this Mainland Chinese cohort of HIV-infected MSM, suicidal ideation may be a transient phenomenon experienced initially following HIV diagnosis, but resolved with increased knowledge and external social support. The results of this research have direct implications for healthcare delivery and HIV counseling, testing and treatment in Mainland China. Public health campaigns are needed to increase support services and develop routine mental health screening and counseling in clinical and community HIV testing and treatment centers. Furthermore, intervention strategies of intentional activities and public health campaigns targeting stigma reduction should also be adopted to facilitate resilience.

Acknowledgements Authors wish to thank all participants who shared their life histories and experiences, Shenzhen 258 Rainbow Workgroup and Shenzhen CDC who helped significantly in the fieldwork and access to the populations. Dr. Joseph T. F. Lau from The Chinese University of Hong Kong provided advice in the original ethnographic study. Dr. Conall O'Cleirigh from Harvard Medical School provided significant assistance in coding mental health symptoms. The publication's contents are solely the responsibility of the authors and do not necessarily represent the official view of the centers or the schools.

Funding This research was funded by the Center for Health Behaviors Research of the Jockey Club School of Public Health and Primary Care (JCSPHPC), The Chinese University of Hong Kong (CUHK), a postgraduate study Grant in JCSPHPC, and the Global Scholarship Programme for Research Excellence-CNOOC Grants 2010-2011 in CUHK.

\section{Compliance with Ethical Standards}

Conflict of interest The authors declare that they have no conflict of interest.

Ethical Approval Ethics approval was granted by the Survey and Behavioral Research Ethics Committee at the Chinese University of Hong Kong.

\section{References}

Apinundecha, C., Laohasiriwong, W., Cameron, M. P., \& Lim, S. (2007). A community participation intervention to reduce HIV/AIDS stigma, Nakhon Ratchasima province, northeast Thailand. AIDS Care, 19, $1157-1165$.

Bertolote, J. M., Fleischmann, A., De Leo, D., \& Wasserman, D. (2003). Suicide and mental disorders: Do we know enough? British Journal of Psychiatry, 183, 382-383.

Bletzer, K. V. (2007). Identity and resilience among persons with HIV: A rural African American experience. Qualitative Health Research, 17, $162-175$.

Boulay, M., Tweedie, I., \& Fiagbey, E. (2008). The effectiveness of a national communication campaign using religious leaders to reduce HIV-related stigma in Ghana. African Journal of AIDS Research, 7, 133-141.

Cadell, S., Karabanow, J., \& Sanchez, M. (2001). Community, empowerment, and resilience: Paths to wellness. Canadian Journal of Community Mental Health, 20, 21-35.

Capron, D. W., Gonzalez, A., Parent, J., Zvolensky, M. J., \& Schmidt, N. B. (2012). Suicidality and anxiety sensitivity in adults with HIV. AIDS Patients Care and STDs, 26, 298-303. 
Carrico, A. W., Johnson, M. O., Morin, S. F., Remien, R. H., Charlebois, E. D., Steward, W. T., ... NIMH Healthy Living Project Team. (2007). Correlates of suicidal ideation among HIV positive persons. AIDS, 21, 1199-1203.

Carrico, A. W., Neilands, T. B., \& Johnson, M. O. (2010). Suicidal ideation is associated with HIV transmission risk in men who have sex with men. Journal of Acquired Immune Deficiency Syndrome, 54, e3-e4. doi:10. 1097/QAI.0b013e3181da1270.

Catalan, J., Harding, R., Sibley, E., Clucas, C., Croome, N., \& Sherr, L. (2011). HIV infection and mental health: Suicidal behavior-Systematic review. Psychology, Health and Medicine, 16, 588-611.

Cattell, V.(2001). Poor people, poor places, and poor health: The mediating role of social networks and social capital. Australian Journal of Social Issues, 39, 329-342.

Chen, G., Li, Y., Zhang, B., Yu, Z., Li, X., Wang, L., \& Yu, Z. (2012). Psychological characteristics in high-risk MSM in China. BMC Public Health, 12, 58. http://www.biomedcentral.com/1471-2458/12/58

Christiansen, E., \& Stenager, E. (2009). Risk for attempted suicide in children and youths after contact with somatic hospitals: A Danish register based nested case-control study. Journal of Epidemiology and Community Health, 66, 247-253.

Devries, K., Watts, C., Yoshihama, M., Kiss, L., Schraiber, L. B., Deyessa, N., \& WHO Multi-Country Study Team. (2011). Violence against women is strongly associated with suicide attempts: Evidence from the WHO Multi-Country Study on Women's Health and Domestic Violence against Women. Social Science and Medicine, 73, 79-86.

Durkheim, E. (1951). Suicide: A study in sociology. New York, NY: Free Press.

Francis, C., Pirkis, J., Dunt, D., Blood, R., \& Davis, C. (2002). Improving mental health literacy: A review of the literature. Melbourne, Australia: Centre for Health Program Evaluation, The University of Melbourne.

Fredrickson, B. L. (2000). Cultivating positive emotions to optimize health and well-being. Prevention and Treatment, 3, 0001a. doi:10.1037/1522-3736. 3.1.31a.

Gibbs, L. M., Dombrovski, A. Y., Morse, J., Siegle, G. J., Houck, P. R., \& Szanto, K. (2009). When the solution is part of the problem: Problem solving in elderly suicide attempters. International Journal of Geriatric Psychiatry, 24, 1396-1404.

Green, J., \& Thorogood, N. (2009). Qualitative methods for health research (2nd ed.). London: Sage.

Hanssens, L. (2010). 'Echo clusters'-Are they a unique phenomenon of indigenous attempted and completed suicide? Aboriginal and Islander Health Worker Journal, 34, 17-26.

Hawton, K., Rodham, K., Evans, E., \& Weatherall, R. (2002). Deliberate self harm in adolescents: Self report survey in schools in England. British Medical Journal, 325, 1207-1211.

Johnson, J., Gooding, P., Wood, A. M., \& Tarrier, N. (2010). Resilience as positive coping appraisals: Testing the Schematic Appraisals Model of Suicide (SAMS). Behaviour Research and Therapy, 48, 179-186.

Johnson, J., Wood, A. M., Gooding, P., Taylor, P. J., \& Tarrier, N. (2011). Resilience to suicidality: The buffering hypothesis. Clinical Psychology Review, 31, 563-591.

Keiser, O., Spoerri, A., Brinkhof, M. W. G., Hasse, B., Gayet-Ageron, A., Tissot, F., ... for the Swiss HIV Cohort Study and the Swiss National Cohort. (2010). Suicide in HIV-infected individuals and the general population in Switzerland, 1988-2008. American Journal of Psychiatry, 167, 143-150.

Kessler, R. C., Borges, G., \& Walters, E. E. (1999). Prevalence of and risk factors for lifetime suicide attempts in the National Comorbidity survey. Archives of General Psychiatry, 56, 617-626.

Kleinman, A., \& Kleinman, J. (1997). Moral transformations of health and suffering in Chinese society. In A. M. Brandt \& P. Rozin (Eds.), Morality and health (pp. 101-118). New York: Routledge.

Lesage, A. D., Boyer, R., Grunberg, F., Vanier, C., Morissette, R., MenardButeau, C., \& Loyer, M. (1994). Suicide and mental disorders: A case- control study of young men. American Journal of Psychiatry, 151, $1063-1068$.

Li, H. C., Holroyd, E., \& Lau, J. T. F. (2010a). Negotiating homosexual identities: The experiences of men who have sex with men in Guangzhou. Culture, Health, and Sexuality, 12, 401-414.

Li, H. C., Holroyd, E., \& Lau, J. T. F. (2015a). Exploring unprotected anal intercourse among newly diagnosed HIV positive men who have sex with men in China: An ethnographic study. PLoS ONE, 10, e0140555. doi:10.1371/journal.pone.0140555.

Li, H. C., Holroyd, E., Lau, J. T. F., \& Li, X. (2015b). Stigma, subsistence, intimacy, face, filial piety, and mental health problems among newly HIV-diagnosed men who have sex with men in China. Journal of the Association of Nurses in AIDS Care, 26, 454-463.

Li, L., Liang, L. J., Lin, C., Wu, Z., \& Rotheram-Borus, M. J. (2010b). NIMH Collaborative HIV/STD Prevention Trial Group. HIV prevention intervention to reduce HIV-related stigma: Evidence from China. AIDS, 24, 115-122.

Li, X., Xiao, Z., \& Xiao, S. (2009). Suicide among the elderly in mainland China. Psychogeriatrics, 9, 62-66.

Masten, A. S. (2001). Ordinary magic: Resilience processes in development. American Psychologist, 56, 227-238.

McCance, T. V., McKenna, H. P., \& Boore, J.R.P.(2001). Exploring caring using narrative methodology: An analysis of the approach. Journal of Advanced Nursing, 33, 350-356.

McNamara, P. M. (2013). Adolescent suicide in Australia: Rates, risk and resilience. Clinical Child Psychology and Psychiatry, 18, 351-369.

Meel, B. L., \& Leenaars, A. A. (2005). Human immunodeficiency virus (HIV) and suicide in a region of Eastern Province ("Transkei"), South Africa. Archives of Suicide Research, 9, 69-75.

Osman, A., Gutierrez, P. M., Muehlenkamp, J. J., Dix-Richardson, F., Barrios, F. X., \& Kopper, B. A. (2004). Suicide resilience inventory-25: Development and preliminary psychometric properties. Psychological Reports, 94, 1349-1360.

Perry, S., Jacobsberg, L., \& Fishman, B. (1990). Suicidal ideation and HIV testing. Journal of the American Medical Association, 263, 679-682.

Phillips, M. (2010). Rethinking the role of mental illness in suicide. American Journal of Psychiatry, 167, 731-733.

Phillips, M., Li, X., \& Zhang, Y. (2002). Suicide rate in China: 1995-1999. Lancet, 359, 835-840.

Pitts, M., Smith, A., Mitchell, A., \& Patel, S. (2006). Private lives: A report on the health and wellbeing on GLBTI Australians. Melbourne: Australian Research Centre in Sex, Health and Society, La Trobe University.

Plena, P., Singer, H., \& Goldbeck, L. (2011). Traumatic events and suicidality in a German adolescent community sample. Journal of Traumatic Stress, 24, 121-124.

Rabkin, J. G., Remien, R., Katoff, L., \& Williams, J. B. W. (1993). Resilience in adversity among long-term survivors of AIDS. Hospital and Community Psychiatry, 44, 162-167.

Riala, K., Ilomaki, E., Hakko, H., \& Rasanen, P. (2011). Is the severity of adolescent conduct disorder associated with the level of nicotine dependence? European Child and Adolescent Psychiatry, 20, 393-399.

Rodgers, B. L., \& Cowles, K. V. (1993). The qualitative research audit trail: A complex collection of documentation. Research in Nursing and Health, 16, 219-226.

Rutter, P. A., Freedenthal, S., \& Osman, A. (2008). Assessing protection from suicidal risk: Psychometric properties of the suicide resilience inventory. Death Studies, 32, 142-153.

Ryan, C., Huebner, D., Diaz, R. M., \& Sanchez, J. (2009). Family rejection as a predictor of negative health outcomes in white and Latino lesbian, gay, and bisexual young adults. Pediatrics, 123, 346-352.

Samuelsson, M., Wiklander, M., Asberg, M., \& Saveman, B. I. (2000). Psychiatric care as seen by the attempted suicide patient. Journal of $A d v$ anced Nursing, 32, 635-643.

Scutella, R., \& Wooden, M. (2008). The effects of household joblessness on mental health. Social Science and Medicine, 67, 88-100. 
Sheldon, K. M., \& Lyubomirsky, S. (2006). Achieving sustainable gains in happiness: Change your actions, not your circumstances. Journal of Happiness Studies, 7, 55-86.

Shenzhen City Government General Office. (2011). The 12th five years Shenzhen City population development plan. Retrieved on 30 October 2011 from http://www.sz.gov.cn/zfgb/2011/gb758/201110/t2011101 2_1738467.htm

Sherr, L., Lampe, F., Fisher, M., Arthur, G., Anderson, J., Zetler, S., \& Harding, R. (2008). Suicidal ideation in UK HIV clinic attenders. AIDS, 22, 1651-1658.

Siegel, K., \& Meyer, I. H. (1999). Hope and resilience in suicide ideation and behavior of gay and bisexual men following notification of HIV infection. AIDS Education and Prevention, 11, 53-64.

Sin, N. L., \& Lyubomirsky, S. (2009). Enhancing well-being and alleviating depressive symptoms with positive psychology interventions: A practice-friendly meta-analysis. Journal of Clinical Psychology, 65, 467-487.

Smith, M. K., McCarragher, T. M., \& Brown, G. T. (2015). Struggles and resilience of African American women living with HIV or AIDS: A qualitative study. Journal of Social Work, 15, 409-424.

Starace, F., \& Sherr, L. (1998). Suicidal behaviours, euthanasia and AIDS. AIDS, 12, 339-347.
Thompson, E. (2002). The Lazarus phenomena: An exploratory study of gay men living with HIV. Social Work in Health Care, 37, 87-114.

van Heeringen, C., Bijttebier, S., \& Godfrin, K. (2011). Suicidal brains: A review of functional and structural brain studies in association with suicidal behavior. Neuroscience and Biobehavioral Reviews, 35, 688-698.

Weich, S., Patterson, J., Shaw, R., \& Stewart-Brown, S. (2009). Family relationships in childhood and psychiatric disorders in later life: A systematic review of prospective studies. British Journal of Psychiatry, 194, 392-398.

Wilcox, H. C., Conner, K. R., \& Caine, E. D. (2004). Association of alcohol and drug use disorders and completed suicide: An empirical review of cohort studies. Drug and Alcohol Dependence, 76(Suppl. 7), S11S19.

Wu, F. (2009). Suicide and justice. London: Routledge.

Wu, F. (2011). Suicide, a modern problem in China. In A. Kleinman, Y. Yan, J. Jun, S. Lee, E. Zhang, P. Tianshu, et al. (Eds.), Deep China: The moral life of the person: What anthropology and psychiatry tell us about China today? (pp. 213-236). Berkeley, CA: University of California Press.

Xie, W., Chen, L., Cai, W., Liu, G., Zhao, J., \& Tan, J. (2010). Estimation of the population size of men who have sex with men in Shenzhen in 2010. Chinese Journal of AIDS and STD, 17, 414-415. 